...blood

biomarkers

of immune

changes in

response to

the drug were

also observed

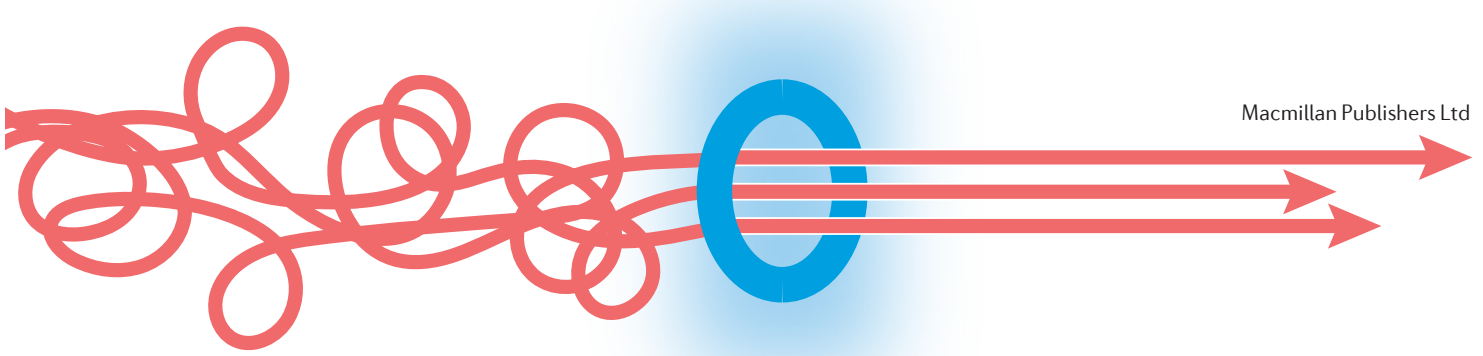

$\Rightarrow$ THERAPY

\title{
Immunotherapy advance for T1DM
}

Therapies that modulate the immune system of patients with type 1 diabetes mellitus (T1DM) have been the focus of much research in the past few years; however, no therapy that is both efficacious and safe has been identified so far. New research published in Science Translational Medicine presents promising early findings for an immunotherapy using an HLA-DR4 $\left(D R B 1^{\star} 0401\right)$-restricted immunodominant proinsulin peptide.

This phase Ib placebo-controlled trial included 27 adult patients who had been diagnosed with T1DM in the previous 100 days. The participants were randomly assigned to receive placebo, the proinsulin peptide every 2 weeks for 6 months or the proinsulin peptide every 4 weeks for 6 months. Patients were followed up for 12 months after initiation of therapy to assess blood biomarkers of response, tolerability and safety. "Safety is an important issue for immunotherapy in T1DM, which has a delicate equipoise," explains corresponding author Mark Peakman.

The treatment was found to be well tolerated, with no systemic or local hypersensitivity or serious adverse effects. Importantly, blood biomarkers of immune changes in response to the drug were also observed. "Both of these findings are quite consistent with what is known from similar studies in other diseases, but this is new for T1DM," says Peakman.

As expected, patients who received the placebo experienced a steady decline in levels of stimulated C-peptide (a marker of insulin secretion) and a $50 \%$ increase in daily insulin use over the follow-up period. By contrast, levels of stimulated C-peptide and insulin use did not change in either intervention group.
The proinsulin peptide seems to modify the $\mathrm{T}$ cell response, as treated patients demonstrated increased IL-10 production and increased FOXP3 expression by regulatory $\mathrm{T}$ cells.

Although these are very positive findings, the study is limited by a small sample size. "We obviously need a well-powered study to look at efficacy - can we change the course of progressive loss of $\beta$-cell function in T1DM?" says Peakman. “The implications of the current paper are yes, we can proceed safely to do that, and with a well-tolerated drug maybe now we can also include children in our next steps, which is where a lot of the disease burden is."

Claire Greenhill

ORIGINAL ARTICLE Alhadj Ali, M. et al. Metabolic and immune effects of immunotherapy with proinsulin peptide in human new-onset type 1 diabetes. Sci. Transl Med. http://dx.doi. org/10.1126/scitranslmed.aaf7779 (2017) 